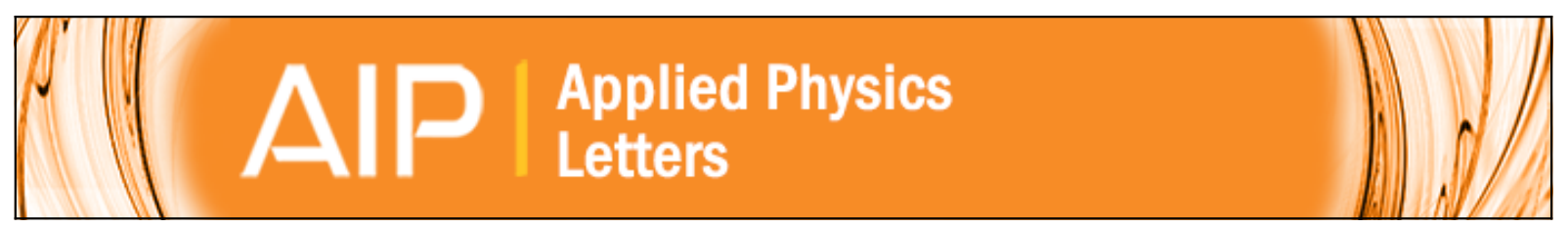

\title{
Mechanical anomaly impact on metal-oxide-semiconductor capacitors on flexible silicon fabric
}

M. T. Ghoneim, A. Kutbee, F. Ghodsi Nasseri, G. Bersuker, and M. M. Hussain

Citation: Applied Physics Letters 104, 234104 (2014); doi: 10.1063/1.4882647

View online: http://dx.doi.org/10.1063/1.4882647

View Table of Contents: http://scitation.aip.org/content/aip/journal/apl/104/23?ver=pdfcov

Published by the AIP Publishing

\section{Articles you may be interested in}

Reliability properties of metal-oxide-semiconductor capacitors using LaAIO 3 high- $\mathrm{k}$ dielectric

Appl. Phys. Lett. 95, 162902 (2009); 10.1063/1.3250242

Gate oxide scaling down in $\mathrm{Hf} \mathrm{O} 2$ - Ga As metal-oxide-semiconductor capacitor using germanium interfacial passivation layer

Appl. Phys. Lett. 91, 042904 (2007); 10.1063/1.2762291

Temperature-induced voltage drop rearrangement and its effect on oxide breakdown in metal-oxidesemiconductor capacitor structure

J. Appl. Phys. 97, 044504 (2005); 10.1063/1.1850199

Gamma-ray-irradiation effects on the leakage current and reliability of sputtered TiO 2 gate oxide in metal-oxide-semiconductor capacitors

J. Appl. Phys. 91, 9198 (2002); 10.1063/1.1473668

Localization of gate oxide integrity defects in silicon metal-oxide-semiconductor structures with lock-in IR thermography

J. Appl. Phys. 88, 4000 (2000); 10.1063/1.1310185

You don't

still use this

cell phone

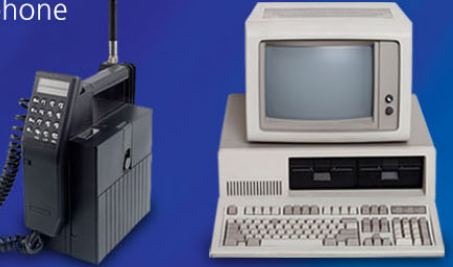

Why are you still using an AFM designed in the 80 's?
It is time to upgrade your AFM

Minimum \$20,000 trade-in discount for purchases before August 31st

Asylum Research is today's technology leader in AFM 


\title{
Mechanical anomaly impact on metal-oxide-semiconductor capacitors on flexible silicon fabric
}

\author{
M. T. Ghoneim, ${ }^{1}$ A. Kutbee, ${ }^{1}$ F. Ghodsi Nasseri, ${ }^{2}$ G. Bersuker, ${ }^{3}$ and M. M. Hussain ${ }^{1, a)}$ \\ ${ }^{1}$ Integrated Nanotechnology Lab, Electrical Engineering, Computer Electrical Mathematical Science and \\ Engineering, King Abdullah University of Science and Technology, Thuwal 23955-6900, Saudi Arabia \\ ${ }^{2}$ The KAUST Schools (TKS), 4700 King Abdullah University of Science and Technology, Thuwal 23955-6900, \\ Saudi Arabia \\ ${ }^{3}$ SEMATECH, 257 Fuller Road, Albany, New York 12203, USA
}

(Received 11 April 2014; accepted 28 May 2014; published online 10 June 2014)

We report the impact of mechanical anomaly on high- $\kappa /$ metal-oxide-semiconductor capacitors built on flexible silicon (100) fabric. The mechanical tests include studying the effect of bending radius up to $5 \mathrm{~mm}$ minimum bending radius with respect to breakdown voltage and leakage current of the devices. We also report the effect of continuous mechanical stress on the breakdown voltage over extended periods of times. (C) 2014 AIP Publishing LLC. [http://dx.doi.org/10.1063/1.4882647]

Flexible electronics are an emerging field extending from stylish product design to new opportunities for traditional electronics. It opens up new, previously not feasible application areas, such as advanced healthcare devices, which can be attached to the body for monitoring vital signs. ${ }^{1-9}$ A variety of approaches have been explored in quest of high flexibility of electronic devices without sacrificing the advantages of traditional devices.

The mainstream technologies to fabricate flexible electronics can be divided into three main categories. The first one is fabricating organic electronic devices on naturally flexible polymeric substrates, which has the advantages of low cost and excellent flexibility. However, these devices usually exhibit low mobility inherent to organic materials, low integration density, and mandated process temperature limitations to avoid substrate melting. ${ }^{10-12}$ The second technique involves fabrication of the inorganic devices on a regular silicon substrate followed by a device transfer to a flexible polymeric substrate. ${ }^{13-17}$ In this approach, the advantages of the polymeric substrates flexibility and good performance of inorganic devices are preserved. This technique is mostly focused on emerging applications, and the work of achieving the integration density needed for highperformance computational electronics is still ongoing. The third approach is to capitalize on the semiconductor industry's expensive and abrasive fabrication processes (epitaxy, high energy implantation, spalling, abrasive back grinding, etc.) and the resultant product has limited bendability and no transparency. ${ }^{18,19}$ To complement these efforts, we have previously reported a complementary metal oxide semiconductor (CMOS) compatible process using trench-protect-releaserecycle process to transform conventionally fabricated bulk electronics into the flexible and semi-transparent one. This versatile process can be applied to any type of CMOS devices fabricated on regular Si (100) wafers. As was previously reported, the variety of devices on our flexible Si platform exhibits no significant degradation comparing to the conventional bulk devices. ${ }^{20-25}$

\footnotetext{
${ }^{\text {a) }}$ Author to whom correspondence should be addressed. Electronic mail: muhammadmustafa.hussain@kaust.edu.sa.
}

In this work, we assess the impact of mechanical anomaly (bending radius, bending cycles, and hold time at a certain bending condition) on the characteristics of the metaloxide-semiconductor capacitors (MOSCAPs) representing a basic element of electronic circuits built on our flexible $\mathrm{Si}$ platform.

We fabricated high- $\kappa$ metal gate MOSCAP devices on $300 \mathrm{~nm}$ thermally grown $\mathrm{SiO}_{2}$ on $\mathrm{Si}$ (100) bulk 4" wafer. The process is depicted in Figure 1. First, we pattern the field oxide (FOX) to layout the active area. Then, we deposit the gate stack composed of $10 \mathrm{~nm}$ high- $\kappa\left(\varepsilon_{\mathrm{r}}=\mathrm{C} * \mathrm{t} / \varepsilon_{0} \mathrm{~A} \sim 6.8\right)$ $\mathrm{Al}_{2} \mathrm{O}_{3}$ dielectric deposited by atomic layer deposition (ALD) followed by $20 \mathrm{~nm}$ of ALD tantalum nitride (TaN) without breaking the vacuum. Finally, aluminum $(\mathrm{Al})$ contacts are made for probing the devices. To this end, the fabrication of

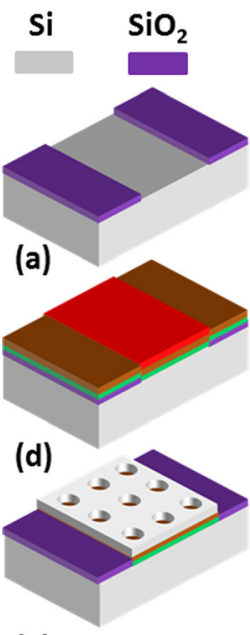

(g)

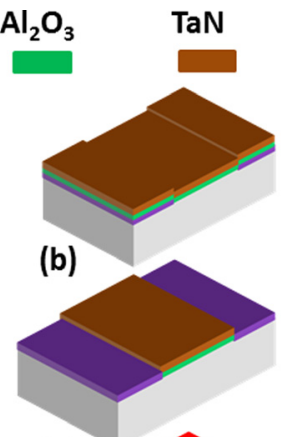

(e)

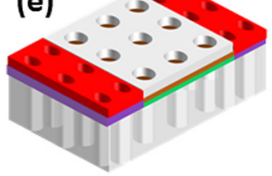

(h)

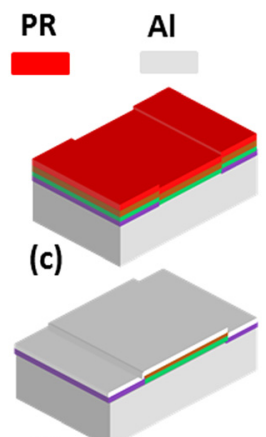

(f)

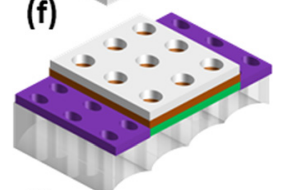

(i)
FIG. 1. Fabrication process of flexible MOSCAPs: (a) patterning of active area, (b) ALD deposition of $10 \mathrm{~nm} \mathrm{Al}_{2} \mathrm{O}_{3} / 20 \mathrm{~nm} \mathrm{TaN}$, (c) and (d) protecting active area using photoresist, (e) etching ALD stack for isolation using RIE and stripping PR, (f) physical vapor deposition of $200 \mathrm{~nm}$ of Al contacts, (g) patterning the $\mathrm{Al}$ electrodes including release holes, (h) patterning release holes across sample and deepening them using Bosch DRIE process to etch $25 \mu \mathrm{m}$ Si trenches followed by stripping of photoresist then, using spacer technique, protecting cylindrical sidewalls (not shown) followed by (i) final isotropic release step by etching in $\mathrm{XeF}_{2}$. 
bulk MOSCAPs is completed, and the next step is transforming the silicon wafer into flexible silicon fabric with fabricated devices. This is done by embedding a network of holes into the substrate using a contact mask and subsequent hole etching. The etch was performed using a deep reactive ion etching (DRIE) and the Bosch process of successive etching and deposition steps to obtain vertical smooth sidewalls. Afterwards, $50 \mathrm{~nm}$ of $\mathrm{ALD} \mathrm{Al}_{2} \mathrm{O}_{3}$ is deposited followed by a vertical etch through this film, which leaves the sidewalls of the holes protected similar to that of the spacer technique. Then, the sample is placed in a $\mathrm{XeF}_{2}$ etching chamber, where $\mathrm{Si}$ is etched isotropically. Hence, the surface of the sample is partially covered by the Al layers as depicted in Figure 1(a), while the sidewalls of the deep holes are protected too. Therefore, $\mathrm{Si}$ is accessible to $\mathrm{XeF}_{2}$ only at the bottom of the deep holes resulting in a lateral etching of the $\mathrm{Si}$ substrate that enables the peeling off of a thin flexible and semi-transparent Si fabric containing the MOSCAPs (Fig. 2).

Both flexible and inflexible MOSCAPs were characterized by $\mathrm{CV}$ measurements at low and high frequencies using E4980A Precision LCR Meter (Agilent Technologies) with a $30 \mathrm{mV}$ small signal superimposed on bias voltage. Figure 3 shows the plots for the ratios of the flexible/inflexible CV data at different frequencies for the (a) actual MOSCAPs $(10 \mathrm{~nm}$ $\mathrm{Al}_{2} \mathrm{O}_{3}$ ) and (b) parasitics MOSCAPs $(300 \mathrm{~nm} \mathrm{SiO}$, for parasitics extraction), which is used to extract parasitics. The capacitance measured in $100 \times 100 \mu \mathrm{m}^{2}$ devices is normalized per unit area; the flexible devices have $10 \mu \mathrm{m}$ diameter holes with $20 \mu \mathrm{m}$ pitch (i.e., $80 \%$ of the effective area of bulk devices). Comparing the plots in (a) and (b), we notice that for the released parasitics MOSCAPs with $300 \mathrm{~nm} \mathrm{SiO}_{2}$, there is a slight degradation due to etched holes, while for the actual MOSCAPs with $10 \mathrm{~nm} \mathrm{Al}_{2} \mathrm{O}_{3}$, the degradation is significant pointing to a higher density of trapped charges in ALD $\mathrm{Al}_{2} \mathrm{O}_{3}$ compared to that of the thermal oxide. The effect of these trapped charges is even more pronounced in the flexed devices due to their structural modification by the network of release holes. This is because of the processing steps and the new dielectric/air interface from the inside of the release holes. To quantify the effect, the interface defect density $\left(D_{i t}\right)$ for the flexible and inflexible devices is calculated as follows:

$$
\mathrm{D}_{\mathrm{it}}=\frac{\mathrm{C}_{\mathrm{ox}}}{\mathrm{q}^{2}}\left(\frac{\mathrm{C}_{\mathrm{lf}}}{\mathrm{C}_{\mathrm{ox}}-\mathrm{C}_{\mathrm{lf}}}-\frac{\mathrm{C}_{\mathrm{hf}}}{\mathrm{C}_{\mathrm{ox}}-\mathrm{C}_{\mathrm{hf}}}\right)
$$

where $\mathrm{C}_{\mathrm{ox}}$ is the oxide capacitance (capacitance in accumulation region), $\mathrm{q}$ is the elementary electron charge $\left(1.6 \times 10^{-19} \mathrm{C}\right), \mathrm{C}_{\mathrm{lf}}$ and $\mathrm{C}_{\mathrm{hf}}$ are the low frequency and high
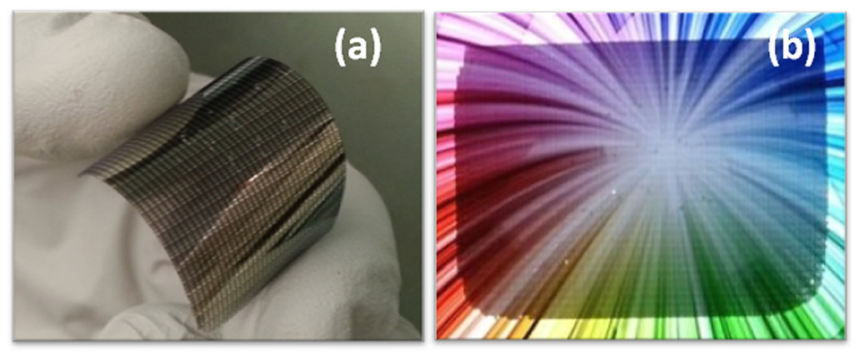

FIG. 2. Digital images showing the flexibility and transparent nature of the flexible Si fabric containing the devices.
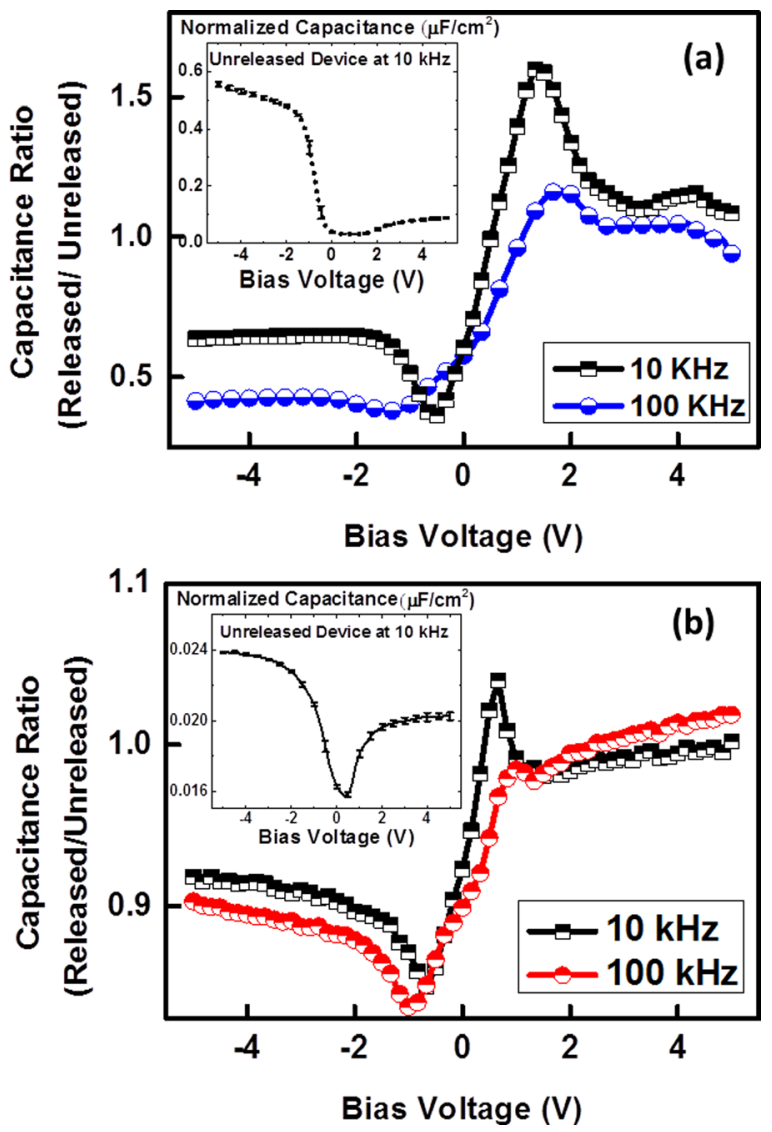

FIG. 3. Capacitance ratio (flexible/inflexible) for (a) actual MOSCAPs and (b) parasitics MOSCAPs $10 \mathrm{kHz}$ and $100 \mathrm{kHz}$, insets showing the normalized capacitance for inflexible devices at $10 \mathrm{kHz}$.

frequency capacitances, respectively, in the depletion-inversion region. Based on Eq. (1), $D_{i t}$ of the released flexible devices is increased by $137 \%$ than that of the inflexible ones. Since both have $\mathrm{Al}_{2} \mathrm{O}_{3}$ as the dielectric, then the increase in $\mathrm{D}_{\mathrm{it}}$ can be attributed to the structural features associated with the presence of a network of release holes.

Since the reported, here, flexible devices have been fabricated using a conventional process flow and structure, we focus on assessing the mechanical anomaly related performance analysis of these devices. Specifically, we study the effect of external mechanical strain at different bending radii and time of strain at specific bending angles on the breakdown voltage of the flexible MOSCAP devices. This is especially important since in many biomedical applications, for example, to monitor vital signs of body organs, the flexible devices are actually bent most of the time.

Figure 4(a) shows a variation in the average breakdown voltage with different bending radius corresponding to varying a nominal strain $\left(\varepsilon_{\text {nominal }}=\mathrm{t} / 2 \mathrm{R}, \mathrm{t}\right.$ is the substrate thickness and $\mathrm{R}$ is the bending radius), the inset depicting the bending measurements test structures. The plot shows that the average breakdown voltage increases with the increased applied strain, reaching a 200\% increase at $\varepsilon_{\text {nominal }}$ of $0.25 \%$. This was observed in the past too as self-healing property of $\mathrm{Al}_{2} \mathrm{O}_{3}$, where $\mathrm{Al}_{2} \mathrm{O}_{3}$ thin films exhibit lower leakage currents on subsequent sweeps and explained by an electrical anneal phenomenon. ${ }^{26}$ In our case, more stressed bonds (due to higher strain at lower 

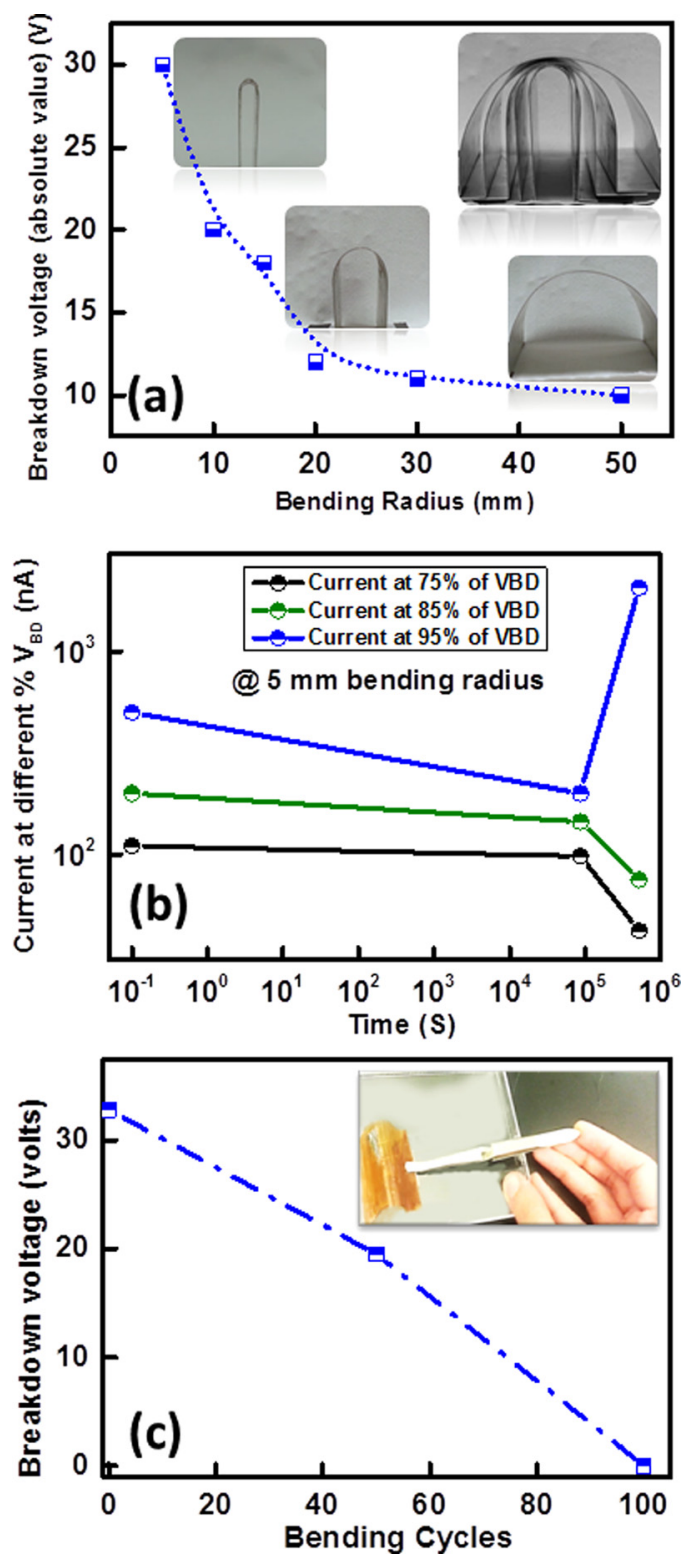

FIG. 4. (a) Change of average breakdown voltage of MOSCAPs versus bending radius; (b) Leakage current versus time corresponding to different values of constant stress voltages; (c) Breakdown voltage as a function of bending cycles. Inset: the samples are extended using the Kapton tape from both edges to enable manual bending of the sample at the center of the bending structure.

bending radius) initially pass higher currents leading to a similar electrical annealing step which heals the device and makes it less prone to breakdown.

Another important indication of the reliability of flexible devices is the leakage current, which have been monitored during the extended periods of time. Figure 4(b) shows the leakage current in flexible MOSCAPs at different stress voltages, as a percentage of the average breakdown voltage, over a time period of 6 days $\left(5 \times 10^{5} \mathrm{~s}\right)$, while being bent at $5 \mathrm{~mm}$ bending radius $\left(\varepsilon_{\text {nominal }}=0.25 \%\right)$. The trend shown in this plot indicates that the devices can safely operate at $75 \%-85 \%$ of their breakdown voltage for a little more than a day. The observed leakage current decrease over stress time is attributed to the significant charge trapping, which is especially more prominent in $\mathrm{Al}_{2} \mathrm{O}_{3}$ high- $\kappa$ dielectric. On the other hand, operation at $95 \%$ of breakdown voltage shows an order of magnitude increase in leakage current, which is indicative of a permanently damaged dielectric in a time dependent dielectric breakdown (TDDB) test.

Further, mechanical assessment is performed to determine the durability of these devices when tampered mechanically. This is done by monitoring the breakdown voltage value of the devices after a number of bending cycles at the minimum bending radius of $5 \mathrm{~mm}$ corresponding to $0.25 \%$ nominal strain. The bending steps were done manually to simulate a real environment where the devices are exposed to physical unbalanced perturbation due to an expanding heart, for instance.

Figure 4(c) shows the resulting breakdown voltage versus the number of bending manual cycles showing around $12 \%$ decrease in breakdown voltage after 50 cycles and complete failure after 100 cycles. This imposes a limitation on these flexible MOSCAPs as they cannot withstand multiple flexing and de-flexing cycles making them unsuitable for applications where frequent bending at $5 \mathrm{~mm}$ bending radius is required. But this is due to bending cycles, as we have reduced the bending radius to $2 \mathrm{~mm}$ and after 80 cycles the devices are physically broken. We also noticed the same phenomena when we tested the fabric at $5 \mathrm{~mm}$ bending radius where getting closer to 100 cycles the fabric shows fracture. To overcome this limited mechanical endurance issue, we believe the fabric will need polymeric support which will be necessary during final device packaging. Such support will increase the mechanical endurance of flexible silicon based devices.

In this paper, we report the mechanical strain impact on the high- $\kappa /$ metal gate MOSCAPs built on widely used bulk mono-crystalline silicon (100) substrates, which was subsequently transformed into the flexible thin $\mathrm{Si}$ fabric. The results show that these devices can withstand higher voltages when bent at smaller bending radii (up to $5 \mathrm{~mm}$ ) for a small time, survive extended time periods under continuous mechanical stress, although can be exposed only to a limited number of bending cycles due to the mechanical fracturing which can be improved by adding polymeric support.

We thank KAUST OCRF Competitive Research Grant (CRG) 1 CRG-1-2012-HUS-008 for supporting this research.

${ }^{1}$ H. Zhou, J.-H. Seo, D. M. Paskiewicz, Y. Zhu, G. K. Celler, P. M. Voyles, W. Zhou, M. G. Lagally, and Z. Ma, Sci. Rep. 3, 1291 (2013).

${ }^{2}$ A. Lecavelier des Etangs-Levallois, M. Lesecq, F. Danneville, Y. Tagro, S. Lepilliet, V. Hoel, D. Troadec, D. Gloria, C. Raynaud, and E. Dubois, Solid-State Electron. 90, 73-78 (2013).

${ }^{3}$ B. Nasr, D. Wang, R. Kruk, H. Rösner, H. Hahn, and S. Dasgupta, Adv. Funct. Mater. 23, 1729 (2013).

${ }^{4}$ S. T. Han, Y. Zhou, C. Wang, L. He, W. Zhang, and V. Roy, Adv. Mater. 25, 793 (2013).

${ }^{5}$ P. Sonar, T. J. Ha, T. R. B. Foong, and A. Dodabalapur, 223rd ECS Meeting, Toronto, Ontario, Canada, 2013.

${ }^{6}$ S. Farsinezhad, A. Mohammadpour, A. N. Dalrymple, J. Geisinger, P. Kar, M. J. Brett, and K. Shankar, J. Nanosci. Nanotechnol. 13, 2885-2891 (2013).

${ }^{7}$ L. A. Majewski, M. Grell, S. D. Ogier, and J. Veres, Org. Electron. 4, 27-32 (2003).

${ }^{8}$ M. C. McAlpine, R. S. Friedman, and C. M. Lieber, Proc. IEEE 93, 1357-1363 (2005).

${ }^{9}$ Y. Sun and J. A. Rogers, Adv. Mater. 19(15), 1897-1916 (2007).

${ }^{10}$ J. N. Tiwari, J. S. Meena, C.-S. Wu, R. N. Tiwari, M.-C. Chu, F.-C. Chang, and F.-H. Ko, ChemSusChem 3(9), 1051-1056 (2010).

${ }^{11}$ M. K. Hota, M. K. Bera, and C. K. Maiti, Semicond. Sci. Technol. 27(10), 105001 (2012). 
${ }^{12}$ A. Bag, M. K. Hota, S. Mallik, and C. K. Maiti, Semicond. Sci. Technol. 28(5), 055002 (2013).

${ }^{13}$ A. Carlson, A. M. Bowen, Y. Huang, R. G. Nuzzo, and J. A. Rogers, Adv. Mater. 24(39), 5284-5318 (2012).

${ }^{14}$ S. A. Stauth and B. A. Parviz, Proc. Natl. Acad. Sci. U.S.A. 103(38), 13922-13927 (2006).

${ }^{15}$ T. Kim, Y. H. Jung, H.-J. Chung, K. J. Yu, N. Ahmed, C. J. Corcoran, J. S. Park, S. H. Jin, and J. A. Rogers, Appl. Phys. Lett. 102(9), 182104 (2013).

${ }^{16}$ C. H. Lee, D. R. Kim, and X. L. Zheng, Proc. Natl. Acad. Sci. U.S.A. 107(22), 9950-9955 (2010).

${ }^{17}$ S. Kim, H. Y. Jeong, S. K. Kim, S.-Y. Choi, and K. J. Lee, Nano Lett. 11(12), 5438-5442 (2011).

${ }^{18}$ D. Shahrjerdi and S. W. Bedell, Nano Lett. 13(1), 315-320 (2012).

${ }^{19}$ D. Shahrjerdi, S. W. Bedell, A. Khakifirooz, K. Fogel, P. Lauro, K. Cheng,

J. A. Ott, M. Gaynes, and D. K. Sadana, Proceedings of IEEE
International Electron Devices Meeting (IEDM), San Francisco, CA, USA, 2012.

${ }^{20}$ J. P. Rojas, G. T. Sevilla, and M. M. Hussain, Appl. Phys. Lett. 102, 064102 (2013).

${ }^{21}$ J. P. Rojas and M. M. Hussain, Phys. Status Solidi RRL 7, 187-191 (2013).

${ }^{22}$ J. P. Rojas, M. T. Ghoneim, C. D. Young, and M. M. Hussain, IEEE Trans. Electron Devices 60, 3305-3309 (2013).

${ }^{23}$ J. P. Rojas, G. A. Torres Sevilla, and M. M. Hussain, Sci. Rep. 3, 2609 (2013).

${ }^{24}$ M. T. Ghoneim, J. P. Rojas, A. M. Hussain, and M. M. Hussain, Phys. Status Solidi RRL 8, 163-166 (2014).

${ }^{25}$ G. T. Sevilla, S. B. Inayat, J. P. Rojas, A. M. Hussain, and M. M. Hussain, Small 9, 3916-3921 (2013).

${ }^{26}$ C.-C. Lu, Y.-C. Lin, C.-H. Yeh, J.-C. Huang, and P.-W. Chiu, ACS Nano 6, 4469-4474 (2012). 